\title{
On universality in human correspondence activity
}

\author{
R. Dean Malmgren ${ }^{1 *}$, Daniel B. Stouffer ${ }^{1,2}$, \\ Andriana S. L. O. Campanharo ${ }^{1,3}$, Luís A. Nunes Amaral ${ }^{1,4 *}$ \\ ${ }^{1}$ Department of Chemical and Biological Engineering, Northwestern University, \\ Evanston, IL 60208, USA \\ ${ }^{2}$ Integrative Ecology Group, Estación Biológica de Doñana, CSIC, 41092 Sevilla, Spain \\ ${ }^{3}$ Instituto Nacional de Pesquisas Espaciais, 12227-010 São José dos Campos, \\ São Paulo, Brazil \\ ${ }^{4}$ Northwestern Institute on Complex Systems, Northwestern University, \\ Evanston, IL 60208, USA \\ ${ }^{*}$ To whom correspondence should be addressed; \\ E-mail: dean.malmgren@u.northwestern.edu,amaral@northwestern.edu.
}

Identifying and modeling patterns of human activity has important ramifications in applications ranging from predicting disease spread to optimizing resource allocation. Because of its relevance and availability, written correspondence provides a powerful proxy for studying human activity. One school of thought is that human correspondence is driven by responses to received correspondence, a view that requires distinct response mechanism to explain e-mail and letter correspondence observations. Here, we demonstrate that, like e-mail correspondence, the letter correspondence patterns of 16 writers, performers, politicians, and scientists are well-described by the circadian cycle, task repetition and changing communication needs. We confirm the universality of these mechanisms by properly rescaling letter and e-mail correspondence statistics to reveal their underlying similarity.

Power-law statistics are a hallmark of critical phenomena. A less obvious characteristic of crit- 
icality is the emergence of universality classes that capture the similarity of seemingly disparate systems. For example, despite the fact that water and carbon dioxide have different chemical properties, they were observed to behave in the same manner close to their respective critical points (1). This is because idiosyncrasies, such as the existence of electric dipoles or the ability to form hydrogen bonds, become irrelevant near the liquid-gas critical point. For physical systems, renormalization group theory $(2,3)$ has enabled researchers to understand the deep connection between the symmetries of a system and the mechanisms which underlie its behavior. The similarity of different fluids near their respective liquid-gas critical points is often demonstrated by rescaling their statistics such that they collapse onto the same universal curves-oftentimes power-laws which have particular scaling exponents. By grouping different substances into the same "universality class," as identified by its scaling exponents, one uncovers that fluids are described by the same statistical laws near the liquid-gas critical point as uniaxial magnets are near their paramagnetic critical point (1). Importantly, one can also differentiate the behavior of these systems from the behavior of polymers near the sol-gel transition, which belong to a different universality class (1).

In addition to critical phenomena, power-law scaling has also been widely reported in biology, economics, and sociology (4-10). Renormalization group theory therefore offers a tantalizing hypothesis for the prevalence of particular power-law scaling exponents in social systems: social systems, in analogy with physical systems, may operate near critical points and can therefore be classified into a small number of distinct universality classes. A heated debate has consequently ensued in the literature concerning the "universality of human systems" (in the statistical physics meaning of the phrase). Is there enough statistical evidence for the asymptotic power-law description of the heavy-tailed distributions reported in human systems (11-14)? Is it reasonable to postulate that social systems, like their physical counterparts $(2,3,15)$, can be classified into universality classes according to scaling exponents (16)?

Human correspondence is a paradigmatic area where the matter of power-law scaling and universality are contentious issues. One view that has recently received significant attention in the literature $(17,18)$ posits that correspondence patterns are driven primarily by the need to respond 
to other individuals. This is formalized by a priority queuing model (19) which, under certain limiting conditions, reproduces the asymptotic scaling of empirically observed heavy-tailed correspondence statistics. In particular, the heavy-tailed statistical properties of e-mail correspondence are reportedly reproduced by a fixed-length queue with a single task type $(19,20)$ whereas the heavy-tailed statistical properties of letter correspondence are reportedly reproduced by either a variable-length queue with a single task type $(20,21)$ or by a fixed-length queue with multiple task types (22). The fact that there are different exponents for the two modes of correspondence has been taken as evidence that human correspondence falls into one of two universality classes (20). When interpreted in the statistical mechanics sense of "universality," one would conclude that email and letter correspondence are fundamentally different activities.

In contrast, we hypothesize that human correspondence patterns are not driven by responses to others but by more prosaic mechanisms_circadian cycles, task repetition and changing communication needs. We formalize these mechanisms with a cascading non-homogeneous Poisson process, which we have previously shown to be statistically consistent with e-mail communication patterns (14). Here, we hypothesize that the same model is capable of describing letter correspondence and that the heavy-tailed correspondence statistics primarily arises from the variation in an individual's communication needs over the course of their lifetime.

We obtained the letter correspondence records for 16 writers, performers, politicians, and scientists. Each data set consists of a list of letters that were sent by each of these individuals, and each record comprises the name of the sender, the name of the recipient, and the date when it was written (see Sec. S1 for details). The nature of the data raises two issues to consider during analysis. First, the precise authorship date of some letters is unknown, so we restrict our analysis to only those letters that have precise authorship dates. Second, it is highly unlikely that all of the letters written by a particular individual are present in the database. We have confirmed that our results are insensitive to sampling effects from this method of data collection (Sec. S2).

An important consideration in studying the letter correspondence patterns of these individuals is that the data covers their entire lifetimes. As a result, it is quite conceivable that changing 
communication needs might affect letter correspondence patterns. For example, before Einstein became widely known, the bulk of his recorded communication was to friends and relatives. After the confirmation of his theory of relativity in 1919, Einstein's need to communicate with other individuals substantially increased. By that time, his step-daughter Ilse Einstein was helping him with secretarial tasks resulting in greatly improved coverage of his recorded correspondence (23). Due to this secretarial assistance and his increased fame, we expect that the average time between consecutively sent letters, the average inter-event time $\langle\tau\rangle$, is significantly larger during the beginning of Einstein's life than during the latter part of his life. Our expectations are verified in Fig. 11A-B, demonstrating that these time series' are non-stationary-that is, the heavy-tailed inter-event time distribution results from a mixture of time scales (24).

Since these time series' are non-stationary, we partitioned each complete time series into smaller time segments so that we can make the approximation that the behavior within each time segment is stationary. We accomplish this by splitting the time series into segments lasting 364 days (52 weeks) unless fewer than 10 events fall within that time period, in which case consecutive segments are merged until this criterion is met.

Assuming that the correspondence patterns within each time segment are stationary, we can then model the behavior within each time segment with standard techniques. As a first approximation, one might naïvely expect that letters are sent at a constant rate $\rho$ and that the time at which every letter is sent is independent of all others. Such a process is referred to as a homogeneous Poisson process, which gives rise to an exponential inter-event time distribution $p(\tau)=\rho e^{-\rho \tau}$. While the tail of the inter-event time distribution within these time segments is approximately exponential, the best-estimate predictions of a homogeneous Poisson process does not produce the correct decay rate (Fig. 1C). This suggests that only a few changes to the homogeneous Poisson process are needed to statistically reproduce the observed inter-event time distribution. We hypothesize that, like e-mail correspondence, two additional ingredients must also be considered for modeling letter correspondence (14).

First, daily and weekly cycles of activity may influence when individuals communicate. Previ- 
ously, we accounted for these cycles of activity in e-mail communication with a non-homogeneous Poisson process whose rate $\rho(t)$ changes periodically on daily and weekly time scales. For letter correspondence, however, the resolution of the data does not permit us to identify activity patterns within a day, and day-to-day changes in activity provide no additional insight (Sec. S3). We therefore approximate the non-homogeneous Poisson process defined by $\rho(t)$ by a homogeneous Poisson process with constant rate $\rho_{i}$ during time segment $i$; that is, we model the rate of activity $\rho(t)$ throughout each individual's life by a piecewise constant function of time.

Second, individuals are much more likely to continue writing letters once they have written one letter in order to use their time more effectively. We account for this behavior by hypothesizing that, once an individual finishes writing a letter, there is a probability $\xi_{i}$ that they write another letter. This process repeats itself until this cascade of additional letters concludes with probability $1-\xi_{i}$, at which point the individual's behavior is again governed by a homogeneous Poisson process with rate $\rho_{i}(25)$. We refer to the resulting model as a cascading Poisson process.

To compare the predictions of the cascading Poisson process (26) to the empirical data, we must first estimate the parameters $\boldsymbol{\theta}_{i}=\left\{\rho_{i}, \xi_{i}\right\}$ from the data during each time segment. The nature of the data, however, raises an important concern for parameter estimation: since each event is only known to occur within a particular day, not at a precise time of the day, the data are interval censored (27). We account for the interval censored data and calculate the best-estimate parameters $\widehat{\boldsymbol{\theta}}_{i}$ by numerically maximizing the censored likelihood function (see Sec. S4 for the derivation).

The resulting best-estimate parameters $\widehat{\boldsymbol{\theta}}_{i}$ provide insight into the correspondence patterns of each individual (Fig. 2A-B and Fig. S4). For example, while both Schoenberg and Einstein have a 50-fold increase in the rate at which the send letters-presumably due to their increasing correspondence obligations and a more complete sampling of their overall letter correspondence — their utilization of cascades of activity is markedly different. Schoenberg, for instance, sends about $21 \%$ of his letters during cascades of multiple letters throughout his life. In contrast, Einstein rarely utilizes cascades of activity as a young man (before 1910) whereas in later years (after 1933) he sends approximately $34 \%$ of his letters during cascades of multiple letters. 
In the period 1928-1933, Einstein sent over 50\% of his letters during cascades of multiple letters. The start of this period coincides with the hiring of Einstein's long-time secretary Helen Dukas, who more systematically retained copies of his outgoing correspondence. After the Nazis took over power in January 1933, his correspondence patterns change markedly; this possibly reflects changes in his correspondence obligations at Princeton University after immigrating to the United States in late 1933 (23).

Of course, inferring how an individual's behavior changes based on a model's parameter estimates is contingent upon the model being consistent with the data. We tested the statistical consistency of our model with the data by Monte Carlo hypothesis testing (Sec. S5). We reject the model during a particular time segment if the $p$-value obtained from the Monte Carlo hypothesis testing procedure is less than a threshold of 0.05 . Because this threshold is greater than zero, it means that there is a finite chance that we will reject the hypothesis that the model is consistent with the data even if the data was generated from the model.

If we assume that each time segment is independent, then we would expect to reject each of the time segments with a $5 \%$ chance and the total number of rejections to be distributed according to a binomial model (28). Out of the 54 independent time segments for Einstein for example, we would expect to reject the model 2.7 times with 0-6 defining the bounds of the $95 \%$ confidence interval of the corresponding binomial model. For Einstein, our procedure "rejects" the cascading Poisson process for 2 out of 54 time segments, indicating that we cannot reject the hypothesis that the model is able to explain his correspondence patterns. Indeed, our hypothesis testing confirms that the cascading Poisson process can not be rejected as an explanatory model for the letter correspondence of any of the individuals under consideration (Tbl. 1). These results demonstrate that the origin of the heavy-tailed inter-event time distribution is a mixture of distributions with different time scales (Fig. 2C-E).

Our findings enable us to address a crucial question: do e-mail and letter correspondence belong to different universality classes (20)? Since the same mechanistic model is capable of describing both e-mail and letter correspondence, we can answer this question in the negative. We 
demonstrate the underlying similarity of both correspondence activities by rescaling and collapsing the inter-event time distributions for 16 randomly selected e-mail correspondents (29) for which we have model parameter estimates (14) and the 16 letter correspondents studied here (Fig. 3). The rescaled inter-event time distributions agree with theoretical expectations (30), demonstrating that the same exponential statistical law is indeed capable of describing both correspondence patterns.

Only by understanding and validating the underlying mechanisms can we appropriately rescale e-mail and letter correspondence to reveal their underlying similarity. Unlike critical phenomena, the universality here does not arise from the irrelevance of idiosyncrasies but rather from the fact that these two different modes of communication are governed by the same mechanisms. This insight is not apparent just by studying the asymptotic scaling of an empirical distribution obtained from an individual; one simply cannot infer that different "scaling" exponents necessarily imply different mechanisms.

Our results therefore raise significant questions about the nature of universality in complex phenomena, in general, and in human correspondence, in particular. Perhaps the most common universal statistical law is due to the central limit theorem-sums of variates with finite fluctuations converge to a Gaussian distribution. When confronted with statistical patterns that are non-Gaussian one is tempted to surmise that the system's fluctuations are not finite. In analogy to physical systems, the recurrence of power-law dependencies with similar exponent values in biological or social systems is frequently hypothesized to arise from the fact that these systems operate near critical points where particular details of the system become irrelevant.

A less-explored hypothesis, as exemplified here, is that heavy-tailed distributions emerge as a result of non-stationarities in the absence of criticality $(14,31)$. Our study demonstrates that human correspondence can be accurately modeled as a cascading non-homogeneous Poisson process-a non-critical process. This process gives rise to heavy-tailed statistics but not to power-law statistics characterized by critical exponents. Instead, the correspondence patterns of each individual are uniquely characterized by the parameters of our model (32); the process is universal, but the parameters are not. 
Indeed, we postulate that the cascading Poisson process, which formally incorporates the circadian cycle, task repetition and changing needs, may accurately describe many other aspects of human activity. The circadian cycle has such physiologic impact that it is natural to surmise that it will affect numerous human behaviors, from eating habits to commuting routines. Task repetition is similarly important due to the increased efficiency it enables; once an individual makes one purchase at a mall, it is easier to make other purchases within that mall during the same trip than it is to return to the mall the following day. As one ages and changes roles, it is not hard to imagine that the extent with which the circadian cycle and task repetition influence their activity might change over time. It is therefore plausible that the cascading Poisson processes presented here could be generalized to account for different types of activities, each with its own evolving parameters.

\section{References and Notes}

1. H. E. Stanley, Introduction to Phase Transitions and Critical Phenomena (Oxford Univeristy Press, Oxford, 1971).

2. S.-K. Ma, Modern Theory of Critical Phenomena (Addison-Wesley Publishing Co., Redwood City, CA, 1976).

3. N. D. Goldenfeld, Lectures on Phase Transitions and the Renormalisation Group (AddisonWesley, Reading, MA, 1992).

4. L. Bachelier, Annales Scientifiques de l'École Normale Supérieure 3, 21 (1900).

5. V. Pareto, Manuale di Economia Politica (Milano, Societa Editrice, 1906).

6. F. Auerbach, Petermanns Geographische Mitteilungen 59, 74 (1913).

7. R. Gibrat, Les Inégalités Économiques (Librairie du Recueil Sirey, Paris, 1931). 
8. G. K. Zipf, Human Behavior and the Principle of Least Effort: An Introduction to Human Ecology (Addison-Wesley Press, Cambridge, MA, 1949).

9. Y. Ijiri, H. A. Simon, Skew Distributions and the Sizes of Business Firms, vol. 24 of Studies in Mathematical and Managerial Economics (North-Holland, Amsterdam, Netherlands, 1977).

10. M. E. J. Newman, Contemp. Phys. 46, 323 (2005).

11. D. Avnir, O. Biham, D. Lidar, Science 279, 39 (1998).

12. L. A. N. Amaral, A. Scala, M. Barthélémy, H. E. Stanley, Proc. Natl. Acad. Sci. USA 97, 11149 (2000).

13. A. Clauset, C. R. Shalizi, M. E. J. Newman, Power-law distributions in empirical data. arXiv:0706.1062.

14. R. D. Malmgren, D. B. Stouffer, A. E. Motter, L. A. N. Amaral, Proc. Natl. Acad. Sci. USA 105, 18153 (2008).

15. H. E. Stanley, L. A. N. Amaral, P. Gopikrishnan, V. Plerou, Physica A 283, 31 (2000).

16. H. E. Stanley, et al., Physica A 224, 302 (1996).

17. C. Castellano, S. Fortunato, V. Loreto, Statistical physics of social dynamics. arXiv:0710.3256.

18. T. Zhou, X.-P. Han, B.-H. Wang, Towards the understanding of human dynamics. arXiv:0801.1389v1.

19. A.-L. Barabási, Nature 435, 207 (2005).

20. A. Vázquez, et al., Phys. Rev. E 73, 036127 (2006).

21. J. G. Oliveira, A.-L. Barabási, Nature 437, 1251 (2005).

22. G. Grinstein, R. Linsker, Phys. Rev. Lett. 97, 130201 (2006). 
23. T. Sauer (2009). Private communication.

24. J. M. Hausdorff, C.-K. Peng, Phys. Rev. E 54, 2154 (1996).

25. We assume that $\xi_{i} \gg \rho_{i} d t$. This model formulation is equivalent to having $N_{a}$ additional events where $N_{a}$ is drawn from an exponential distribution with mean $\xi_{i} /\left(1-\xi_{i}\right)$.

26. Materials and methods are available as supporting material on Science Online.

27. R. B. D’Agostino, M. A. Stephens, Goodness-of-Fit Techniques (Marcel Kekker, Inc., New York, NY, 1986).

28. R. G. Miller Jr., Simultaneous Statistical Inference (Springer-Verlag, New York, 1991).

29. J.-P. Eckmann, E. Moses, D. Sergi, Proc. Natl. Acad. Sci. USA 101, 14333 (2004).

30. H. M. Taylor, S. Karlin, An Introduction to Stochastic Modeling (Academic Press, San Diego, CA, 1994).

31. L. A. N. Amaral, S. V. Buldyrev, S. Havlin, M. A. Salinger, H. E. Stanley, Phys. Rev. Lett. 80, 1385 (1998).

32. R. D. Malmgren, J. M. Hofman, L. A. N. Amaral, D. J. Watts, Proceedings of the 15th ACM SIGKDD International Conference on Knowledge Discovery and Data Mining (2009), pp. $607-615$.

33. We thank T. Sauer, R. Guimerà, M. Sales-Pardo, M.J. Stringer, E.N. Sawardecker, J. Duch and P. McMullen for insightful comments and suggestions. D.B.S. acknowledges the support of a CSIC-JAE Postdoctoral Fellowship. A.S.L.O.C. acknowledges the support of a CNPq (Brazil) Doctoral Fellowship. L.A.N.A. gratefully acknowledges the support of NSF award SBE 0624318. All figures were generated with PyGrace (http://pygrace.sourceforge.net) with color schemes from http://colorbrewer.org. 


\section{Supporting Online Material}

www.sciencemag.org

Materials and Methods

Supporting text

Figs. S1-S5

Tbls. S1-S3 
Tbl. 1: Summary of the letter correspondence records and hypothesis testing results for the 16 individuals under consideration, ordered chronologically. For each individual, we note the time period and duration of the letter correspondence records, the total number of letters sent, the number of time segments with at least 10 letters per time segment, the $95 \%$ confidence interval (CI) bounds of the corresponding binomial model with $p=0.05$, and the number of rejections of the cascading Poisson process based on our Monte Carlo hypothesis testing procedure. The number of Monte Carlo hypothesis testing rejections is within the $95 \%$ confidence interval bounds for all 16 individuals, indicating that this model can not be rejected for any individual's letter correspondence patterns. We have conducted the same analysis for three alternative models; we find that a cascading Poisson process provides the most parsimonious and statistically consistent explanation of the data (Sec. S3).

\begin{tabular}{lccrcrc}
\hline & Time & Duration & Number of & Number of & & Number of \\
Individual & Period & $(\mathrm{yr})$ & letters & segments & $95 \%$ CI & rejections \\
\hline Francis Bacon & $1574-1626$ & 53 & 443 & 19 & {$[0,3]$} & 3 \\
James H. Leigh Hunt & $1790-1859$ & 70 & 408 & 25 & {$[0,3]$} & 1 \\
Charles Darwin & $1822-1882$ & 61 & 6,785 & 52 & {$[0,5]$} & 4 \\
Anna Brownell Jameson & $1833-1860$ & 28 & 119 & 8 & {$[0,2]$} & 1 \\
Friedrich Engels & $1833-1895$ & 63 & 369 & 24 & {$[0,3]$} & 1 \\
Robert E. Lee & $1835-1870$ & 36 & 282 & 10 & {$[0,2]$} & 0 \\
Karl Marx & $1837-1882$ & 46 & 469 & 25 & {$[0,3]$} & 1 \\
Henry Irving & $1852-1905$ & 54 & 1,205 & 35 & {$[0,4]$} & 0 \\
Sigmund Freud & $1872-1939$ & 68 & 3,130 & 49 & {$[0,5]$} & 2 \\
Marcel Proust & $1879-1922$ & 44 & 668 & 25 & {$[0,3]$} & 2 \\
H. G. Wells & $1895-1946$ & 52 & 422 & 16 & {$[0,2]$} & 0 \\
Albert Einstein & $1896-1955$ & 60 & 10,319 & 54 & {$[0,6]$} & 2 \\
Carl Sandburg & $1898-1966$ & 69 & 1,894 & 37 & {$[0,4]$} & 2 \\
Arnold Schoenberg & $1902-1951$ & 50 & 6,899 & 47 & {$[0,5]$} & 3 \\
Ernest Hemingway & $1909-1961$ & 53 & 1,934 & 42 & {$[0,5]$} & 5 \\
Stan Laurel & $1924-1964$ & 41 & 685 & 17 & {$[0,3]$} & 1 \\
\hline
\end{tabular}



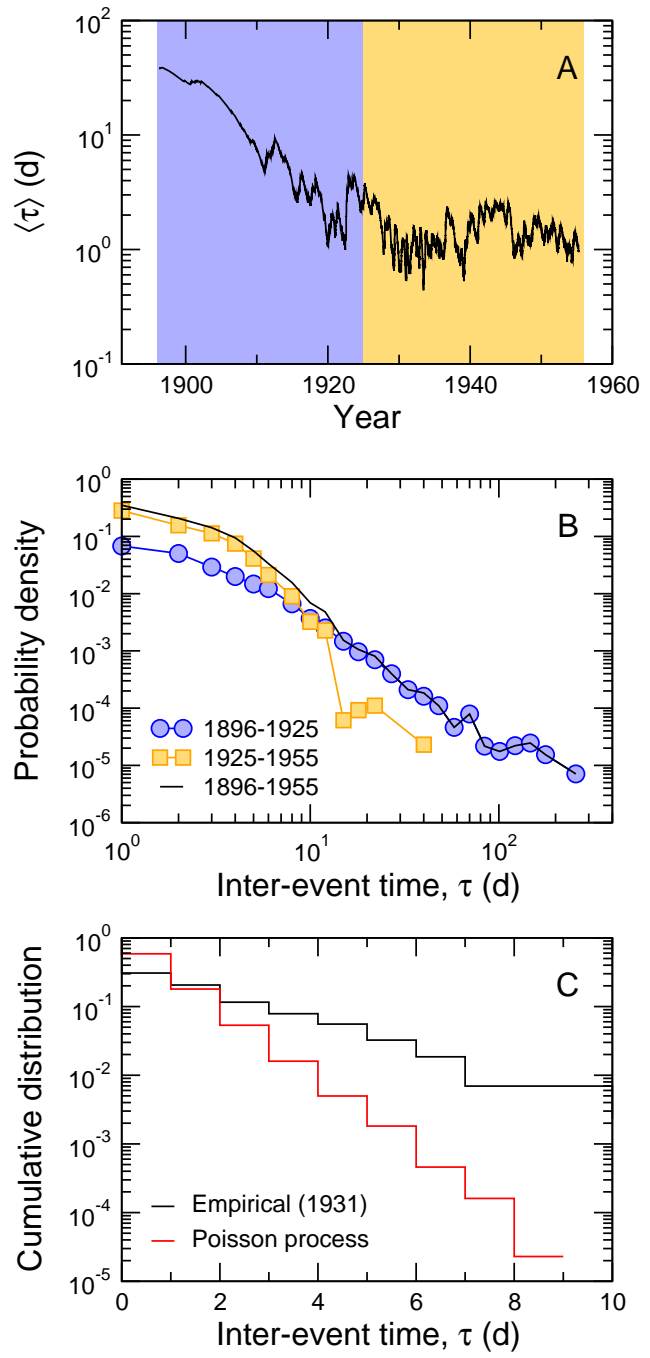

Fig. 1: 
Fig. 1] Non-stationarity of Albert Einstein's letter correspondence activity. While we select Einstein as an example, non-stationarities are present for all 16 writers, performers, politicians, and scientists studied here. A, Running average inter-event time $\langle\tau\rangle$ averaged over 100 consecutive inter-event times. During the beginning of Einstein's life (blue shaded region), the average interevent time is significantly larger than during the end of his life (orange shaded region). B, Logarithmically binned probability density of the non-zero inter-event times $\tau$. If we separately consider the inter-event time distribution during each portion of Einstein's life, it is clear that the complete inter-event time distribution (black line) is actually a mixture of behaviors. To emphasize the origins of the heavy-tailed distribution, the probability densities of each portion of Einstein's life are normalized such that their integrals are equal to the fraction of non-zero inter-event times during that time. C, Comparison of the empirical inter-event time distribution during a particular time segment with the simulated predictions of the best-estimate homogeneous Poisson process that is interval censored in the same manner as the data. It is visually apparent that a homogeneous Poisson process is not consistent with the empirical data, which is confirmed by Monte Carlo hypothesis testing (Sec. S3). 

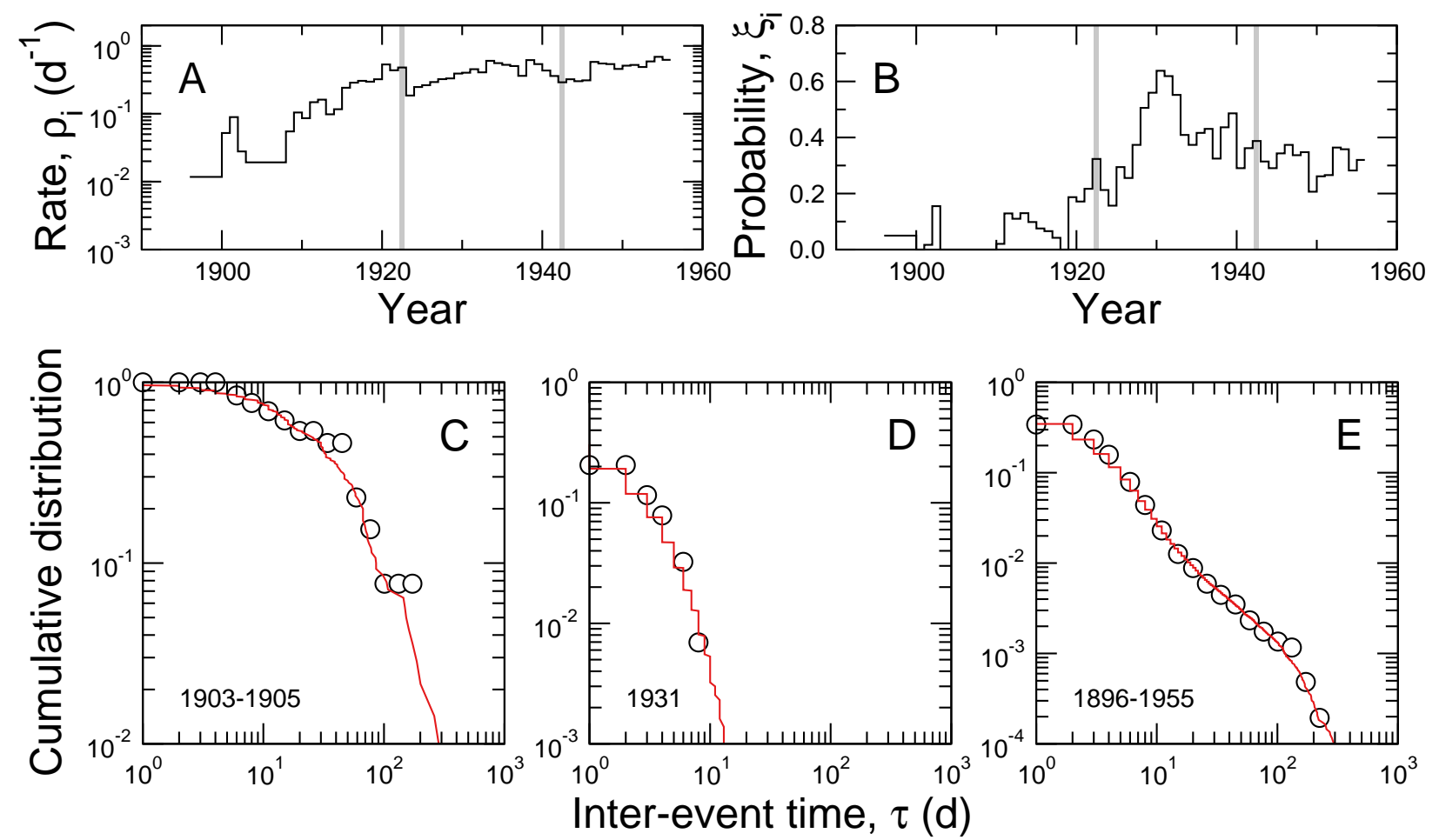

Fig. 2: 
Fig. 2: Origin of heavy-tailed inter-event time distribution for Albert Einstein. While we select Einstein as an example, the same explanation is relevant for all 16 writers, performers, politicians, and scientists studied here. A-B, We estimate the parameters $\boldsymbol{\theta}_{i}=\left\{\rho_{i}, \xi_{i}\right\}$ by maximizing the censored likelihood function for each time segment (Sec. S4). Grey shaded regions denote time segments during which the cascading Poisson process is rejected by Monte Carlo hypothesis testing. Parameter estimates for all individuals under consideration can be found in Fig. S4. Note the 50-fold changes in the rate $\rho_{i}$ and the dramatic changes in $\xi_{i}$ for Einstein. $\mathbf{C}-\mathbf{D}$, The cumulative distribution of inter-event times for Einstein during particular time segments compared with the predictions of a non-stationary cascading Poisson process with the best-estimate parameters (A-B). The model predictions are generated numerically by running the model defined by $\boldsymbol{\theta}(t)$ ten-times and interval censoring the resulting synthetic time series in the same manner as the empirical data. C, The cumulative distribution of inter-event times (circles) for Einstein over his entire life compared with the predictions of a non-stationary cascading Poisson process (red line) with the best-estimate parameters (A-B). When the mixture of behaviors are taken into account, the origin of the heavy-tailed inter-event time distribution is clear. The inter-event time distributions for all 16 letter correspondents under consideration can be found in Fig. S5. 


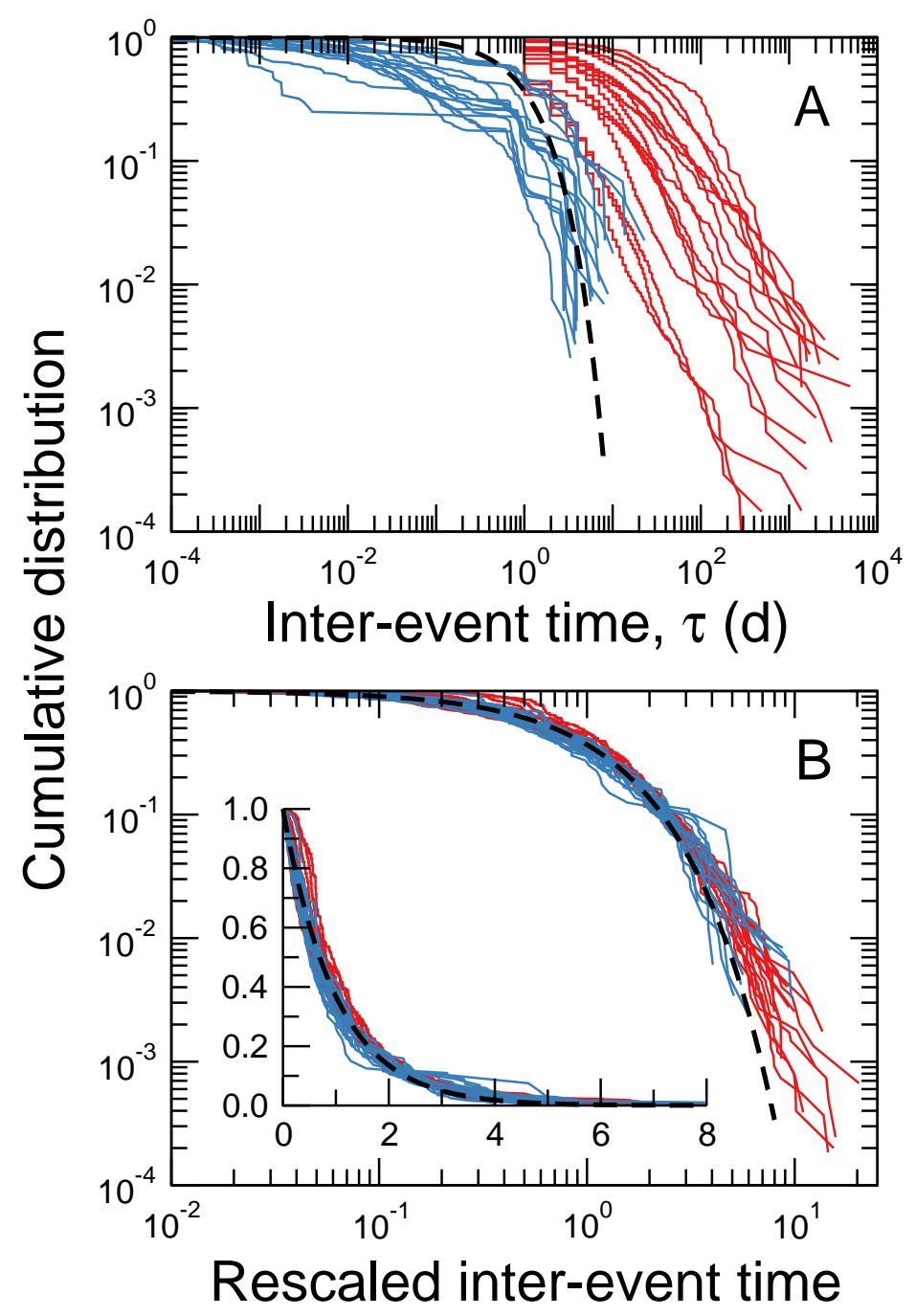

Fig. 3: Collapse of inter-event time distributions for letter and e-mail correspondence. $\mathbf{A}, \mathbf{C u}-$ mulative distribution of inter-event times for all 16 letter correspondents (red lines) and 16 randomly selected e-mail correspondents (blue lines). B, Cumulative distribution of rescaled interevent times on logarithmic and linear (inset) axes. The inter-event time $\tau_{k}=t_{k+1}-t_{k}$ is rescaled by the average inter-event time expected during the interval $\left[t_{k}, t_{k+1}\right]$, which is given by $\langle\tau\rangle=\left(t_{k+1}-t_{k}\right) / \int_{t_{k}}^{t_{k+1}} \rho(s) d s$. By the time rescaling theorem (30), the resulting rescaled inter-event time distribution is given by the expected inter-event time distribution for a homogeneous Poisson process with unit rate $P(\tau)=e^{-\tau}$ (black dashed line). We only consider inter-event times $\tau>0$ for letter correspondence. 